weeks in an uncomplicated six-hour labour and the delivery of three healthy boys. Their birthweights, in birth order, were $1.91 \mathrm{~kg}(4 \mathrm{lb} 4 \mathrm{oz}), 1.77 \mathrm{~kg}$ ( $3 \mathrm{lb}$ $15 \mathrm{oz})$ and $1.77 \mathrm{~kg}$ ( $3 \mathrm{lb} 15 \mathrm{oz})$. No neonatal disorders of consequence were recorded in the maternity book or recollected by the mother.

They have been proven monozygotic by DNA fingerprinting: zygosity, determined by CELLMARK Diagnostics, used the multilocus probes 33.15 and 33.6 on a sample of venous blood from each triplet, and the three sets of $\mathbf{4 8}$ bands identified were identical.

The second-born developed a major depression at 32 years of age, which failed to respond to drugs and psychotherapy. Six months after onset, delusions, hallucinations and passivity experiences occurred which required in-patient care, neuroleptics and ECT. Three years on, medication with neuroleptics and lithium continues to be required to control delusions and depressed mood. Employment has failed. Both siblings remain well apart from intermittent episodes of depression not severe enough to interfere with work.

The mother has a history of intermittent depressive disorder and two episodes of major depression. The father has no psychiatric history; his two siblings are said to have primary cerebellar ataxia.

Magnetic resonance imaging of all three triplets, read blind to the clinical facts, showed the right temporal horn to be larger than the left in the first born sibling, possibly also in the last born and within normal limits in the affected second-born sibling. (MRI sequences: saggital $T_{1} \mathrm{~W}$ and axial $T_{2} \mathrm{~W}$ (proton density and TE 90) spin-echo sequences of the entire brain, amplified by coronal $T_{1} \mathrm{~W}$ inversionrecovery sequences of $5 \mathrm{~mm}$ thickness of the temporal lobes).

These findings are against brain structural differences causing the discordancy for psychosis, unless the abnormalities are protective. The most informative possible explanation is a post-zygotic change in the genome of the affected sibling which may ultimately be identifiable; the most likely is an unknown environmental influence on the developing brain.

This letter is published anonymously to prevent identification of the triplets.

\section{Chloroquine-induced psychiatric complications}

SIR: I would like to draw to the attention of Dr Lovestone that the case reported by him was not the first patient with a true manic episode without confusion in response to chloroquine (Journal, July $1991,159,164-165)$. We have previously reported 30 cases (Bhatia et al, 1988a) (age range 4-36 years; 21 females and 9 males). Organic psychosis was the most common diagnosis (16 cases) followed by paranoid schizophrenia in six, manic-depressive psychosis (MDP) (mania) in two, MDP (depression) in two, anxiety neurosis in two and grand mal seizures in two. When the mode of intake of chloroquine was compared with a control group (the cases who were prescribed chloroquine for malaria but did not develop any psychiatric complication), the patients who took chloroquine with milk were significantly over represented in the study group $(P<0.001)$. The dose, salt of the drug (phosphate or sulphate) and whether the intake of drug was on an empty stomach or after meals, were however, not found to be statistically significant. Headache and sleeplessness were reported significantly more by the patients who developed psychiatric complications. The duration of onset of these complications was two to seven days. Out of 30 cases, 26 recovered within three weeks of discontinuation of chloroquine, whereas the remaining four cases took about 12 weeks to recover. Although six cases were below 12 years of age (Bhatia et al, 1988b), there was no statistically significant difference between the age and sex of cases in study and control groups.

The mechanism by which chloroquine can induce psychosis is still not fully understood. One can draw an analogy with various theories put forward to explain quinacrine psychosis. Engel et al (1947) reported that quinacrine acts as a central stimulant through an unknown mechanism. The hypotheses put forward to explain these psychiatric complications include a drug-induced cholinergic imbalance, prostaglandin-E-antagonism (Malek-Ahmadi, 1981) dopaminergic overactivity and a predisposing factor of glucose-6-phosphate dehydrogenase deficiency (Nasr, 1981). To date, none of these hypotheses have been experimentally evaluated.

Bhatia, M. S., Singhal, P. K. \& Dhar, N. K. (1988a) Psychiatric complications of chloroquine. Annals of National Academy of Medical Sciences (India), 24, 223-228.

$-\frac{1}{2}-(1988 b)$ Chloroquine-induced psychosis. Indian Pediatrics, 25, 258-262.

Engel, G. L., Romano, J. \& Ferris, F. B. (1947) Effect of quinacrine (atabrine) on the central nervous system. Archives of Neurology. 58, 337-339.

Malek-Ahmad, P. (1981) Toxic psychosis following use of quinacrine. Journal of Clinical Psychiatry, 42, 481.

NASR, S. (1981) Toxic psychosis following use of quinacrine. Journal of Clinical Psychiatry, 42, 481.

M. S. ВнатіА

Department of Psychiatry

University College of Medical Sciences

Delhi-110095

India 